\title{
Protective Effect of Ethanolamine on Hepatic Preneoplastic Alterations Induced by the Administration of $\mathrm{N}$-Nitrosodiethylamine in Rats
}

\author{
Hajime Sasaki 1,2 \\ ${ }^{1}$ Department of Nutrition and Life Sciences, Kanagawa Institute of Technology, Atsugi, Japan \\ ${ }^{2}$ Nutritional Research Department, Food Science Research Laboratories, Meiji Co. Ltd., Odawara, Japan \\ Email: sasaki@cco.kanagawa-it.ac.jp
}

How to cite this paper: Sasaki, H. (2017) Protective Effect of Ethanolamine on Hepatic Preneoplastic Alterations Induced by the Administration of $\mathrm{N}$-Nitrosodiethylamine in Rats. Food and Nutrition Sciences, 8 , 936-945.

https://doi.org/10.4236/fns.2017.810067

Received: September 13, 2017

Accepted: October 20, 2017

Published: October 23, 2017

Copyright $\odot 2017$ by author and Scientific Research Publishing Inc. This work is licensed under the Creative Commons Attribution International License (CC BY 4.0).

http://creativecommons.org/licenses/by/4.0/

\begin{abstract}
The effects of exogenously administered ethanolamine (Etn) on the $\mathrm{N}$-nitrosodiethylamine (NDA)-induced formation of hepatic lesions in rats were investigated. Sprague-Dawley rats were intraperitoneally administered NDA (100 $\mathrm{mg} / \mathrm{kg}$ body weight) at 7-day intervals, and the animals were allowed free access to water containing Etn ( 15 or $50 \mathrm{mg} / \mathrm{L}$ ) for 35 days. NDA-induced hepatic lesions were assessed according to the number of nodules detectable on the liver surface, areas of clear cell foci observed on histopathological thin sections, hydroxyproline levels in liver homogenates, and blood biochemical marker levels. Compared with those from control rats that were not administered Etn, livers from Etn-exposed rats had significantly fewer surface nodules and smaller areas of clear cell foci, indicating that Etn prevented or delayed the formation of preneoplastic cell alterations. Hydroxyproline levels in livers were significantly lower in Etn-treated rats, indicating that the chemical prevented the formation of fibrotic alterations. The protective effects of Etn on NDA-induced hepatic lesions were demonstrated by changes in blood biochemical marker levels. These results suggest that Etn can protect against cellular alterations induced by a carcinogenic chemical, possibly by enhancing hepatic phospholipid synthesis.
\end{abstract}

\section{Keywords}

Ethanolamine, $N$-Nitrosodiethylamine, Protection, Hepatic lesion, Cellular Alterations 


\section{Introduction}

Ethanolamine (Etn) is a nutrient synthesized from serine in vivo or supplied from food, and it is metabolized as a substrate for synthesizing phosphatidylethanolamine (PE), one of the major phospholipids constituting the mammalian cell membrane. In most cells, Etn is phosphorylated, after which it combines with 1,2-diacylglycerol to form PE. Synthesized PE is integrated into membranes [1] [2] [3] [4]. In hepatocytes, a second pathway for synthesizing phosphatidylcholine (PC) in addition to the major pathway involving choline combining with 1,2-diacylglycerol exists; specifically, three methyl molecules provided from S-adenosylmethionine are added to the Etn moiety in PE to generate PC [5]. Approximately $30 \%$ of PC in hepatocytes is produced by the secondary pathway [6].

It was reported that Etn accelerates the proliferation of cultured cells, in which the agent promotes PE synthesis concomitantly [7]. It was also reported that Etn enhances the proliferation of hepatocytes in primary culture by potentiating the action of growth factors [8] [9]. Although the mechanism of potentiation is unknown, one evident point is that PE and PC synthesis is apparently associated with cell growth activation by Etn in hepatocytes [10].

Stimulation of cell proliferation by Etn is observed in the regenerating liver after partial hepatectomy, in which PE and PC synthesis is enhanced [8] [11]. In the regenerating liver, cell proliferation is stimulated by growth factors such as HGF and EGF [12] [13]. It is speculated that Etn potentiates the activity of growth factors in regenerating areas. During this activity, reorganization of the newly regenerating liver tissue occurs. In Etn-exposed livers, hepatocyte proliferation is enhanced in regenerating areas of the liver without causing structure distortion.

If the liver is injured by chemical substances, then liver regeneration occurs [14]. In damaged areas in injured livers, both cell proliferation and tissue reorganization proceed. If hepatotoxic chemicals are repetitively administered, injuries are continually induced. In injured areas, hepatocyte proliferation will start each time chemicals are administered before structure reconstruction is completed. Growth factors such as HGF exert a protective effect against the progression of liver injury [15] [16]. This effect might be due to the activation of cell proliferation and metabolism of hepatocytes by growth factors. Growth factors can activate many different metabolic pathways including detoxifying systems such as the antioxidant system, which metabolizes reactive oxygen species to inactive molecules [17].

As Etn activates growth factors, it is speculated that the agent can exert an enhanced protective effect on chemically induced liver injury. This protection might reduce the frequencies of the cycle of damage and restoration. In this paper, the effect of Etn on liver injury induced by repetitive administration of $N$-nitrosodiethylamine (NDA), which is known to induce liver injury with fibrosis, was assessed. 


\section{Materials and Methods}

\subsection{Experimental Animals}

Sprague-Dawley rats (male, 7 weeks old, Charles-River Japan) were maintained under $50 \%$ humidity and a 12-h/12-h light/dark cycle and allowed free access to water and animal diet. The rats were acclimated to the diet and environment for 7 days. Chemical administration was started on the $8^{\text {th }}$ day (Day 0). Animal experiments were approved by the Food Science Institute of Meiji Co. Ltd. and followed the Guide for the Care and Use of Laboratory Animals (National Research Council, USA).

\subsection{Induction of Hepatic Injuries Induced by Chemical Administration and Animal Treatment}

NDA suspended in saline was peritoneally administered at a dose of 100 $\mathrm{mg} / 2 \mathrm{~mL} / \mathrm{kg}$ body weight on Days $0,7,14,21$, and 28 .

Etn $\mathrm{HCl}$ (Wako, Japan) was dissolved in water at a concentration of either 15 or $50 \mathrm{mg} \mathrm{Etn/L} \mathrm{on} \mathrm{Days} 0$ - 34. The average volume of water the rats ingested throughout the experimental period was $23 \mathrm{~mL} /$ day/rat. Based on this volume, the amounts of Etn administered were 0.35 and $1.15 \mathrm{mg}$ Etn/day/rat at Etn concentrations of 15 and $50 \mathrm{mg} / \mathrm{L}$, respectively.

\subsection{Blood Collection and Liver Resection}

Rats were fasted $24 \mathrm{~h}$ before dissection, and blood was collected via the descending aorta. After coagulation, serum was separated by centrifugation and used for biochemical analyses. The liver of each animal was resected after blood collection. The right lobe of the resected liver was dissected into two parts. One part was fixed in $10 \%$ formalin, and the other was frozen at $-80^{\circ} \mathrm{C}$ for further analyses. All sample collections were performed after animals were anesthetized using Nembutal.

\subsection{Biochemical Analysis}

The collected sera were subjected to biochemical analyses to measure alanine aminotransferase (ALT, IU/L), aspartate aminotransferase (AST, IU/L), $\gamma$-glutamyl transferase ( $\gamma$-GTP, IU/L), alkaline phosphatase (ALP, IU/L), and bilirubin $(\mathrm{mg} / \mathrm{dL})$ levels. These biochemical markers were assayed using appropriate colorimetric assay kits (Wako, Japan).

\subsection{Assessments of Hepatic Lesions and Alterations}

The surfaces of both the dorsal and ventral sides of resected livers were photographed. White and round nodules detected on the surfaces of both sides were counted. The right lobe of each resected liver was processed to prepare thin paraffin sections, which were stained using Masson's trichrome. Microscopic fields of stained preparations from all resected livers were randomly selected and digitally photographed. Three photographed fields were analyzed using ImageJ 
software (National Institutes of Health, Bethesda, MD, USA) to assess areas of clear cell foci. The areas of clear cell foci were expressed as relative ratios to the total area of a field.

\subsection{Hydroxyproline Quantification}

Fifty milligrams of the right lobe were hydrolyzed in saturated $\mathrm{HCl}$ for amino acid analysis. Amino acids were quantified using the PTH-amino acid method.

\subsection{Statistical Analysis}

All results were analyzed using Dunnett's test.

\section{Results}

On the liver surface, white and round nodules were found as alterations induced by NDA administration (Figure 1(a)). These nodules were predominantly found in the livers of rats administered NDA alone, whereas nodule counts were significantly lower in Etn-treated rats (Figure 1(b)). These macroscopically observed alterations are considered structures primarily formed due to neoplastic changes in hepatocytes and distortions of hepatic lobes. The results indicate that Etn prevented or delayed these changes.

Cellular alterations were found in histopathological sections. Three types of cellular alterations have been identified in the livers of NDA-administered rats, namely clear cell, basophilic, and acidophilic foci. Among these types of foci, clear cell foci were predominant in the livers of rats in this experiment (Figure 2(a)). Clear cell foci were significantly less numerous in Etn-administered rats than in those only treated with NDA (Figure 2(b)). The microscopically observed cellular alterations are believed to correspond to the inner structures of

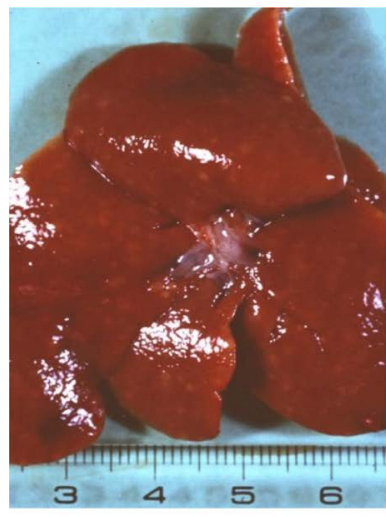

(a)

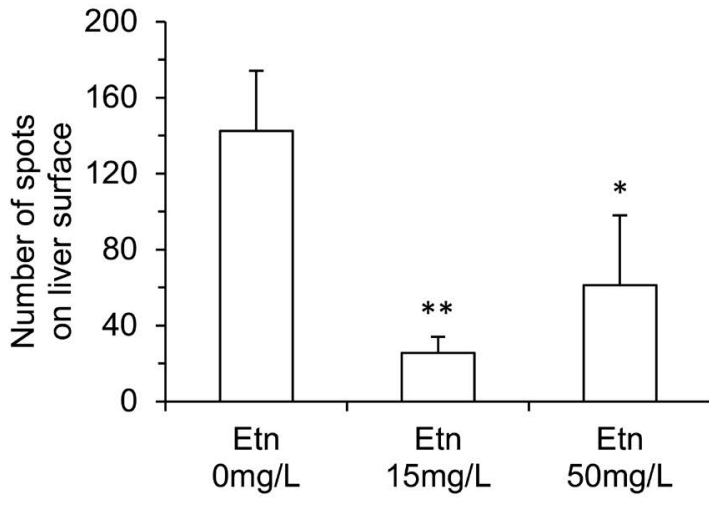

(b)

Figure 1. Nodules on the liver of an NDA-treated rat. (a) White spots were observed located inside the resected liver. The ventral side is shown. The ruler is scales in centimeters. (b) White spots observed through the surface of the resected livers were counted. The total counts on both dorsal and ventral sides were plotted. Rats were administered Etn in drinking water at a concentration of 15 or $50 \mathrm{mg} / \mathrm{L}$. The results are expressed as averages and standard errors. ${ }^{*} p<0.05,{ }^{* *} p<0.01$. 


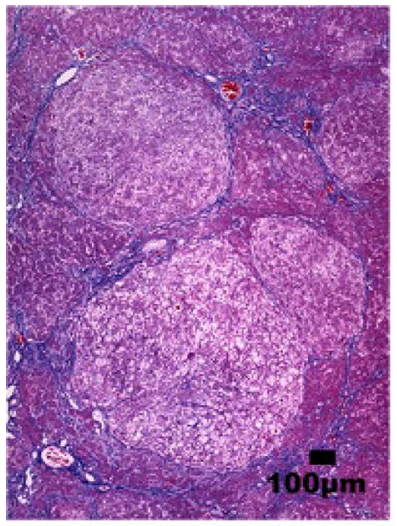

(a)

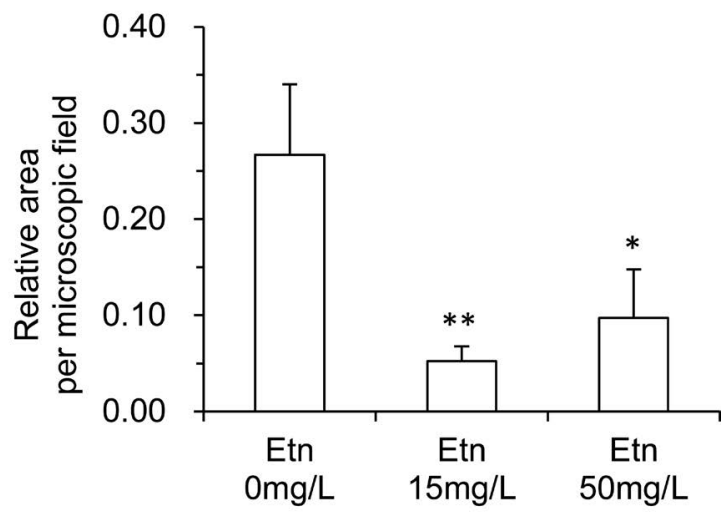

(b)

Figure 2. Histopathological observation of the liver resected from a rat administered $N$-nitrosodiethylamine. (a) Clear cell foci and fibrotic structures at the peripheries of the foci are detected. (b) Areas of clear cell foci on histopathological preparations were measured. Three microscopic fields were randomly selected and photographed. Areas of clear cell foci were calculated using ImageJ. The areas of the foci were converted to ratios relative to the total area of the field. Results are expressed as averages and standard errors. ${ }^{*} p<0.05,{ }^{* *} p<0.01$.

the macroscopically observed alterations on the surfaces of the resected livers. The results indicate that the structural alterations detected by macroscopic observations consisted of neoplastic changes in hepatocytes, which indicate an early stage of tumorigenesis.

The other dominant alteration found on histopathological sections was fibrosis. Structures stained blue on histological sections using Masson's trichrome consist of collagen fibers typical of hepatic fibrosis. Histological sections of livers of NDA-treated rats exhibited blue fibrotic structures surrounding lesions, indicating the development of severe fibrosis (Figure 2(a)).

To quantitatively assess the severity of fibrosis, levels of hydroxyproline, a collagen-specific amino acid, in hydrolyzed liver tissue were measured. Hydroxyproline levels were significantly lower in the livers of Etn-treated rats than in control animals administered only NDA (Figure 3 ). The results indicate that Etn ameliorated NDA-induced fibrosis in a quantitative manner.

Lesions formed in livers were assessed according to blood biochemical marker levels. Levels of AST, which can denote liver injury were lower in rats administered Etn than the control rats that only received NDA (Figure 4), whereas those of ALT were decreased by Etn administration without significance. Meanwhile, those of $\gamma$-GTP, ALP, and bilirubin, which are indicative of biliary dysfunction, were also depressed in Etn-treated rats (Figure 5). Compared to AST levels, which were significantly different depending on the Etn concentration, those of ALT were not affected by the Etn concentration. The low AST/ALT ratio indicates that the biliary lesions were more dominant than hepatocyte destruction [18]. This finding is correlated with the elevated levels of the other enzymes. 


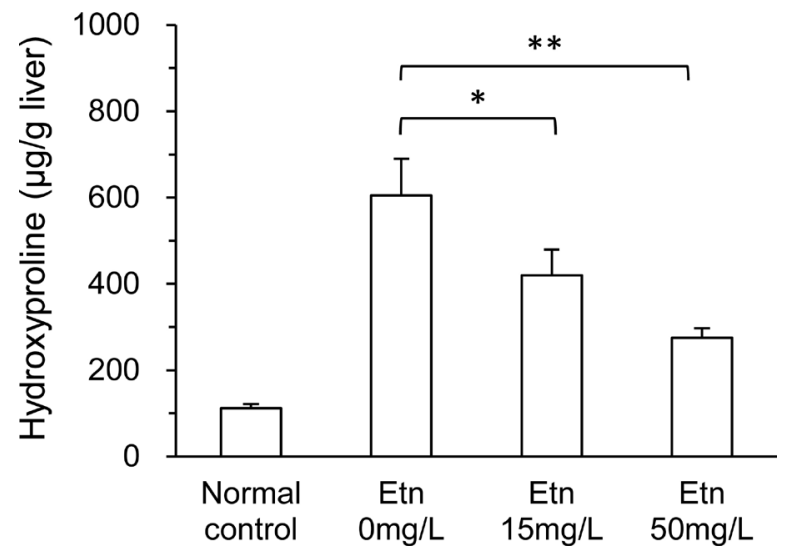

Figure 3. The amounts of hydroxyproline in the homogenates of livers were estimated via amino acid analysis. Amounts were expressed as weights per gram of the liver. Results are expressed as averages and standard errors. ${ }^{\star} p<0.05,{ }^{\star \star} p<0.01$.
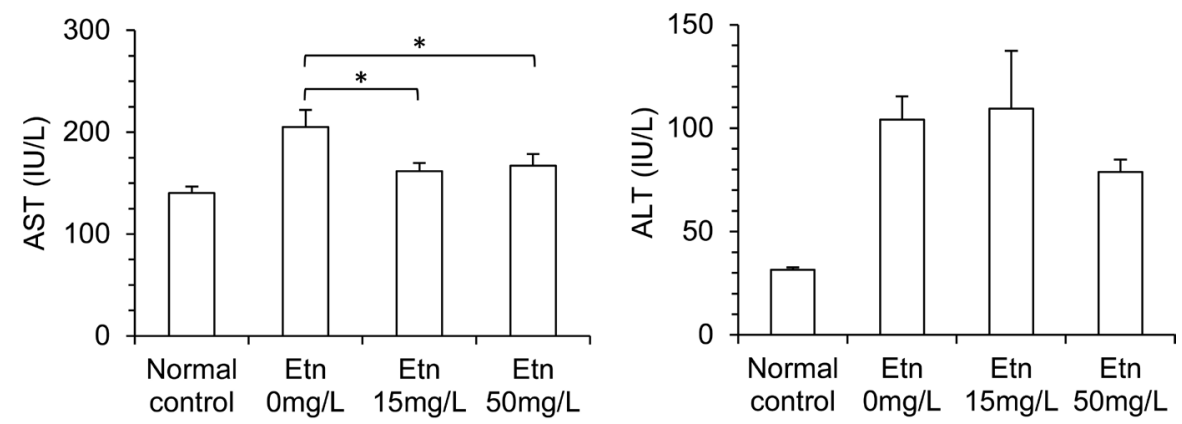

Figure 4. The activities of aspartate aminotransferase (AST) and alanine aminotransferase (ALT) were measured. Results are expressed as averages and standard errors. ${ }^{\star} p<$ 0.05 .
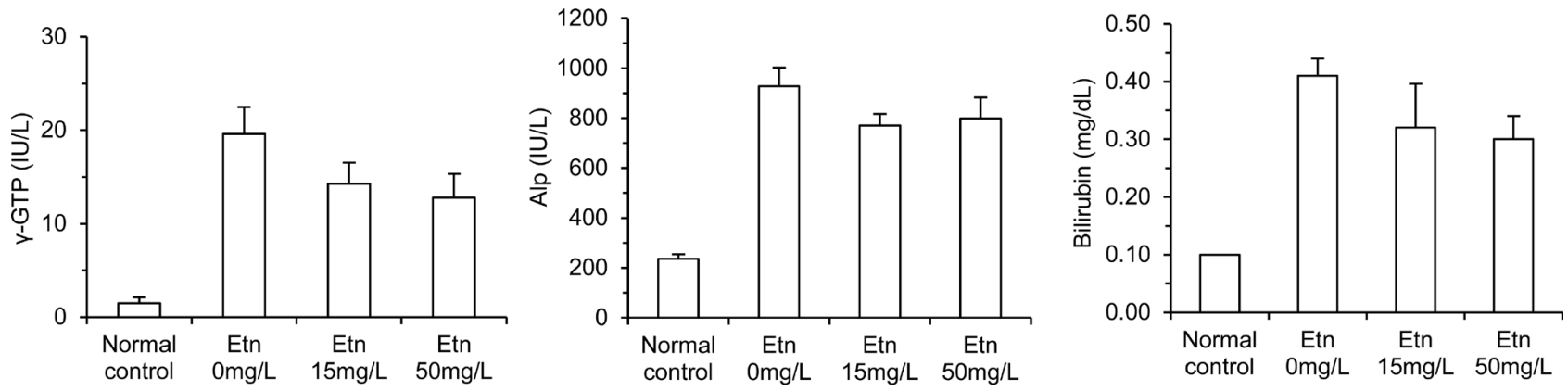

Figure 5. The activities of $\gamma$-glutamyl transferase $(\gamma$-GTP) and alkaline phosphatase (ALP), and the level of bilirubin were measured. Results are expressed as averages and standard errors.

\section{Discussion}

This study described the effects of Etn on hepatic injuries induced by NDA in rats. The major findings were that Etn prevented the formation of preneoplastic lesions, and clear cell foci, and it also prevented the development of fibrosis at the periphery of the foci.

Etn enhances hepatocyte proliferation following hepatectomy [8]. Because Etn enhances PE synthesis in hepatocytes [11], it is speculated that Etn enhances 
hepatocyte proliferation by stimulating phospholipid synthesis. In the livers of NDA-treated rats, the recovery of hepatocytes from damage might be enhanced by the administration of Etn. This speculation might explain the small increases in the levels of AST, a marker of hepatocyte destruction. Contrary to AST levels, ALT levels remained high, and the effects of Etn were not as evident as those on AST. These differing effects of Etn might derive from differences in the sites of damage in the liver. Repetitive NDA administration might induce cycles of hepatocyte damage and recovery. Concomitantly, hepatic lobular structures including biliary structures might be damaged. Damaged hepatocytes might recover by the next NDA insult, whereas damaged biliary structures might not have been reconstructed between cycles of NDA treatment, resulting in progressive structural damage of biliary systems.

The carcinogenic potency of NDA is attributed to its capabilities to induce DNA damage and oxidative stress. NDA can alkylate DNA via cytochrome p450, which damages DNA structures and results in mutated genomic structures [19]. NDA causes oxidative stress by producing reactive oxygen species (ROS). ROS mediates the induction of DNA damage [20] [21], which can result in altered gene expression and carcinogenesis.

Whereas ROS mediates carcinogenesis by damaging DNA, it oxidizes lipids in cell membranes and cytoplasmic proteins, inducing cell death and thus lesion formation [22] [23]. In damaged livers, hepatocytes in areas surrounding lesions start to proliferate and reorganize to restore tissues. If NDA is repetitively administered, hepatocytes in injured areas and their vicinities might be continually damaged, resulting in the formation of chronic lesions. In such tissues, only hepatocytes with mutated DNA might survive and proliferate, resulting in carcinogenesis.

Foci of cellular alterations have been observed in rats in the early stages of chemically induced hepatic carcinogenesis. Principally, three types of foci of cellular alterations have been identified in the livers of NDA-treated rats, specifically clear cell, basophilic, and acidophilic foci [24] [25] [26]. It was reported that clear cell foci become predominant prior to formation of the other foci [25]. It was speculated that clear cell foci are formed by glycogenic cells [24]. Although the details of glycogenic cell formation are unclear, hepatocytes are stimulated to accumulate glycogen following NDA exposure. These hepatocytes are detected as clear cells because cytoplasmic glycogen is washed away during the histopathological preparation process. The predominance of clear cell foci in livers in this report might be due to the administration schedule of NDA.

At the peripheries of clear cell foci, fibrotic structures were observed, as viewed on histopathological preparations (Figure 2(b)). The abundance of fibrotic structures was confirmed by analyzing hydroxyproline levels in livers from non-Etn-exposed rats. In chemical-induced hepatic lesions, pseudolobes are formed when foci of cellular alterations are formed. Pseudolobes consist of foci of cellular alterations on the inside and fibrotic structures at the periphery. 
In Etn-treated rats, clear cell focus formation was suppressed. It is speculated that suppressed formation of fibrotic structures is due to the suppression of focus formation.

Oxidative stress caused by ROS production is rapidly induced in the liver after NDA administration. This stress induces alterations in the antioxidant system. For example, the glutathione S-transferase and aldo-keto reductase superfamilies are upregulated [21]. These machineries function to reduce oxidative stress by converting ROS to unreactive molecules. Oxidative stress can cause hepatic injuries, in which hepatic regeneration occurs as a repair process. In regenerating loci in the liver, hepatocyte proliferation is induced by growth factors. In proliferating hepatocytes, when the machinery for cell proliferation is activated, those for other events such as metabolic reactions including the antioxidant system are also activated. Etn might potentiate the action of growth factors in the regenerating loci, eventually enhancing both hepatocyte proliferation and metabolic reactions. Activation of metabolic reactions will affect the antioxidant system. An activated antioxidant system will reduce ROS levels, thus suppressing oxidative stress.

Etn can act as a cofactor to enhance the activity of hepatocyte-specific growth factors [8]. Etn is a substrate for the synthesis of PE in many types of cells. In hepatocytes, PE is utilized to synthesize PC; specifically, PE is derivatized to PC by the action of the hepatocyte-specific enzyme PE methyltransferase (PEMT) [5]. These phospholipid metabolic pathways in hepatocytes might serve as cofactors to enhance the activity of growth factors. Indeed, after partial hepatectomy, in which hepatocyte regeneration occurs, enhanced PE synthesis is observed. In the meantime, PC synthesis via the PEMT pathway is enhanced. Consequently, a significant increase in the PE ratio was detected [11]. Therefore, enhancement of PE synthesis is a necessary event for hepatocyte proliferation in regenerating livers.

Regarding the requirement of Etn for cell proliferation, cells are classified into two types, Etn-responsive and Etn-nonresponsive. In Etn-responsive cells, Etn enhances both PE synthesis and cell proliferation. In these cells, PE synthesis is a requisite for cell proliferation. Hepatocytes are apparently Etn-responsive [8]. The de novo synthesis of PE that occurs in the presence of lower levels of Etn is not sufficient to enhance hepatocyte function. Higher levels of PE synthesis are necessary to maintain the proliferative and metabolic activities of cells. In regenerating livers, Etn might enhance hepatocyte proliferation concomitantly with enhanced metabolism. Therefore, steady-state levels of Etn might not be sufficient to efficiently activate hepatocyte function in regenerating livers following hepatectomy or NDA treatment. For more efficient hepatocyte function, exogenously administered Etn might be required.

\section{Conclusion}

The results in this report suggest that Etn can protect against cellular alterations induced by the carcinogenic chemical, NDA, presumably by enhancing hepatic phospholipid synthesis. Steady-state levels of Etn might not be sufficient to efficiently activate hepatocyte function in regenerating livers following NDA treat- 
ment. For more efficient hepatocyte proliferation and metabolic activity, exogenously administered Etn might be required.

\section{References}

[1] Bell, R.M. and Coleman, R.A. (1980) Enzymes of Glycerolipid Synthesis in Eukaryotes. Annual Review of Biochemistry, 49, 459-487. https://doi.org/10.1146/annurev.bi.49.070180.002331

[2] Van den Bosch, H. (1974) Phosphoglyceride Metabolism. Annual Review of Biochemistry, 43, 243-277. https://doi.org/10.1146/annurev.bi.43.070174.001331

[3] Tijburg, L., Geelen, M. and van Golde, L.M. (1989) Regulation of the Biosynthesis of Triacylglycerol, Phosphatidylcholine and Phosphatidylethanolamine in the Liver. Biochimica et Biophysica Acta (BBA) - Lipids and Lipid Metabolism, 1004, 1-19. https://doi.org/10.1016/0005-2760(89)90206-3

[4] Zelinski, T.A. and Choy, P.C. (1982) Phosphatidylethanolamine Biosynthesis in Isolated Hamster Heart. Canadian Journal of Biochemistry, 60, 817-823. https://doi.org/10.1139/o82-102

[5] Ridgway, N.D. and Vance, D.E. (1987) Purification of Phosphatidylethanolamine N-Methyltransferase from Rat Liver. The Journal of Biological Chemistry, 262, 17231-17239.

[6] Sundler, R. and Akesson, B. (1975) Regulation of Phospholipid Biosynthesis in Isolated Rat Hepatocytes. Effect of different substrates. The Journal of Biological Chemistry, 250, 3359-3367.

[7] Kano-Sueoka, T., Errick, J.E., King, D. and Walsh, L.A. (1983) Phosphatidylethanolamine Synthesis in Ethanolamine-Responsive and -Nonresponsive Cells in Culture. Journal of Cellular Physiology, 117, 109-115. https://doi.org/10.1002/jcp.1041170115

[8] Sasaki, H., Kume, H., Nemoto, A., Narisawa, S. and Takahashi, N. (1997) Ethanolamine Modulates the Rate of Rat Hepatocyte Proliferation In Vitro and In Vivo. Proceedings of the National Academy of Sciences of USA, 94, 7320-7325. https://doi.org/10.1073/pnas.94.14.7320

[9] Sasaki, H., Nemoto, A., Kume, H., Narisawa, S. and Takahashi, N. (1998) Stimulation of Rat Hepatocyte Proliferation In Vitro and In Vivo by Factors Derived from the Bovine Small Intestinal Mucosa. In Vitro Cellular \& Developmental Biology-Animal, 34, 68-73. https://doi.org/10.1007/s11626-998-0055-4

[10] Kume, H. and Sasaki, H. (2006) Ethanolamine Modulates DNA Synthesis through Epidermal Growth Factor Receptor in Rat Primary Hepatocytes. In Vitro Cellular \& Developmental Biology-Animal, 42, 20-26. https://doi.org/10.1007/s11626-006-0007-9

[11] Kume, H., Sasaki, H. and Kano-Sueoka, T. (2006) Serum Ethanolamine and Hepatocyte Proliferation in Perinatal and Partially Hepatectomized Rats. Life Sciences, 79, 1764-1772.

[12] Michalopoulos, G.K. (2010) Liver Regeneration after Partial Hepatectomy. American Journal of Pathology, 176, 2-13. https://doi.org/10.2353/ajpath.2010.090675

[13] Böhm, F., Köhler, U.A., Speicher, T. and Werner, S. (2012) Regulation of Liver Regeneration by Growth Factors and Cytokines. EMBO Molecular Medicine, 2, 294-305. https://doi.org/10.1002/emmm.201000085

[14] Michalopoulos, G.K. (2007) Liver Regeneration. Journal of Cellular Physiology, 213, 286-300. https://doi.org/10.1002/jcp.21172 
[15] Kaido, T., Yamaoka, S., Seto, S., Funaki, N., Kasamatsu, T., Tanaka, J., Nakamura, T. and Imamura, M. (1997) Continuous Hepatocyte Growth Factor Supply Prevents Lipopolysaccharide-Induced Liver Injury in Rats. FEBS Letters, 411, 378-382.

[16] Okano, J., Shiota, G. and Kawasaki, H. (1997) Protective Action of Hepatocyte Growth Factor for Acute Liver Injury Caused by D-Galactosamine in Transgenic Mice. Hepatology, 26, 1241-1249.

[17] Domínguez-Pérez, M., Nuño-Lámbarri, N., Clavijo-Cornejo, D., Luna-López, A., Souza, V., Bucio, L., Miranda, R.U., Miranda, R.U., Muñoz, L., Gomez-Quiroz, L.E., Uribe-Carvajal, S. and Gutiérrez-Ruiz, M.C. (2016) Hepatocyte Growth Factor Reduces Free Cholesterol-Mediated Lipotoxicity in Primary Hepatocytes by Countering Oxidative Stress. Oxidative Medicine and Cellular Longevity, 2016, Article ID: 7960386.

[18] Giannini, E.G., Testa, R. and Savarino, V. (2005) Liver Enzyme Alteration: A Guide for Clinicians. Canadian Medical Association Journal, 172, 367-379.

https://doi.org/10.1503/cmaj.1040752

[19] Verna, L., Whysner, J. and Williams, G.M. (1996) N-Nitrosodiethylamine Mechanistic Data and Risk Assessment: Bioactivation, DNA-Adduct Formation, Mutagenicity, and Tumor Initiation. Pharmacology \& Therapeutics, 71, 57-81.

[20] Kolaja, K.L. and Klaunig, J.E. (1997) Vitamin E. Modulation of Hepatic Focal Lesion Growth in Mice. Toxicology and Applied Pharmacology, 143, 380-387. https://doi.org/10.1006/taap.1996.8089

[21] Qi, Y., Chen, X., Chan, C.Y., Li, D., Yuan, C., Yu, F., Lin, M.C., Yew, D.T., Kung, H.F. and Lai, L. (2008) Two-Dimensional Differential Gel Electrophoresis/Analysis of Diethylnitrosamine Induced Rat Hepatocellular Carcinoma. International Journal of Cancer, 122, 2682-2688. https://doi.org/10.1002/ijc.23464

[22] Cadet, J. and Wagner, R. (2013) DNA Base Damage by Reactive Oxygen Species. Cold Spring Harbor Perspectives in Biology, 5, pii: a012559. https://doi.org/10.1101/cshperspect.a012559

[23] Jena, N.R. (2012) DNA Damage by Reactive Species: Mechanisms, Mutation and Repair. Journal of Biosciences, 37, 503-517. https://doi.org/10.1007/s12038-012-9218-2

[24] Bannasch, P. (1968) The Cytoplasm of Hepatocytes during Carcinogenesis. Recent Results in Cancer Research, 19, 1-100. https://doi.org/10.1007/978-3-642-88399-6

[25] Moore, M.A., Mayer, D. and Bannasch, P. (1982) The Dose Dependence and Sequential Appearance of Putative Preneoplastic Populations Induced in the Rat Liver by Stop Experiments with N-Nitrosomorpholine. Carcinogenesis, 3, 1429-1436. https://doi.org/10.1093/carcin/3.12.1429

[26] Cortinovis, C., Klimek, F. and Nogueira, E. (1991) Rat Hepatocarcinogenesis Induced by N-Nitrosodiethylamine and N-Nitrosomorpholine Continuously Administered at Low Doses. From Basophilic Areas of Hepatocytes to Hepatocellular Tumors. American Journal of Pathology, 139, 1157-1171. 